\title{
RETRACTED ARTICLE: ASYMMETRIC LEAVES2-LIKE16 gene, a member of AS2/LOB family, is required for lateral root formation of Arabidopsis
}

Guang-Chao Wang $\cdot$ Lai-Sheng Meng •

Xu-Dong Sun $\cdot$ Zhen-Hua Feng $\cdot$ Jian Zhu

Received: 9 December 2009/Revised: 11 December 2009/Accepted: 25 January 2010/Published online: 18 February 2010

(C) Franciszek Górski Institute of Plant Physiology, Polish Academy of Sciences, Kraków 2010

The article is retracted due to disputed authorship and severe omissions in the information of collaborators about the publication.

G.-C. Wang · L.-S. Meng $\cdot$ X.-D. Sun ·

Z.-H. Feng · J. Zhu $(\bowtie)$

School of Life Sciences, Tongji University,

200092 Shanghai, China

e-mail: zhujian1@mail.tongji.edu.cn

L.-S. Meng

e-mail: menglsh2010@yahoo.com.cn 